\title{
The Effect of Melting on Forced Convection Heat Transfer
}

\author{
Chi Tien ${ }^{1}$ and Yin-Chao Yen \\ U. S. Army Cold Regions Research and Engineering Laboratory, Hanover, N. H.
}

(Manuscript received 12 February 1965)

\begin{abstract}
The effect of melting on convective heat transfer between a melting body and surrounding fluid was studied quantitatively from the point of view of boundary layer theory, film theory and penetration theory. These studies indicate that melting retards the rate of heat transfer, and the decrease in heat transfer coefficient is found to be a unique function of the parameter $C_{p} \Delta T / \Delta H_{m}$ where $\Delta T$ is the temperature difference between the fluid and melting body, $C_{p}$ is the heat capacity of the fluid and $\Delta H_{m}$ the enthalpy change due to melting.
\end{abstract}

\section{Introduction}

In the study of the melting rate of a solid body surrounded by warm fluid, such as a drifting iceberg in sea water, an accurate prediction of the heat transfer coefficient between the solid and fluid is of the utmost importance since the transfer coefficient is the critical factor which determines the rate of energy exchange. Although there have been numerous investigations on the convective heat transfer between a solid and fluid under various conditions, most of the studies do not take into account the interfacial velocity resulting from melting. As demonstrated in the study of mass transfer (Bird et al., 1960; Stewart, 1950), the interfacial velocity could greatly disturb the final velocity and temperature profiles and significantly influence the values of transfer coefficient.

In an earlier study, the effect of melting on heat transfer was studied by the present authors (Yen and Tien, 1963) for the Leveque problem. The tangential velocity profile is assumed to be linear. This approximation is valid if one deals with a high Prandtl number fluid so that the significant temperature change takes place only within a thin layer of fluid immediately adjacent to the solid boundary and consequently the velocity profile inside this thin layer can be approximated by a linear segment. Furthermore, the flow was assumed to be laminar. Because of these restrictions, it may not be justified to extend the results to more complicated situations.

The purpose of the present investigation is to study further the effect of melting on convective heat transfer rate. For the case of laminar flow, the two-dimensional equations of motion and energy are solved exactly. In order to extend the study to other conditions such as natural convection heat transfer or forced convective

${ }^{1}$ Present address: Department of Chemical Engineering, Syracuse University, Syracuse, N. Y. heat transfer under turbulent conditions, the effect of melting on heat transfer was also examined on the basis of two familiar theories (film theory and penetration theory) which have been used extensively in mass transfer processes. Although the quantitative results based on each of these theories are different, they all exhibit the same qualitative trend and indicate that under certain conditions, the effect of melting can be very significant.

\section{Symbols}

A dimensionless parameter defined as $C_{p}\left(T_{\infty}-T_{0}\right) /$ $\Delta H_{m}$

$C_{p} \quad$ heat capacity of fluid

E energy flux vector

$f \quad$ a quantity defined by Eq. (9)

$H$ enthalpy of fluid

$H_{0} \quad$ enthalpy of fluid at temperature $T_{0}$ (or $T_{m}$ ) per unit mass

$\Delta H_{m}$ enthalpy change due to melting

$h_{m} \quad$ heat transfer coefficient with melting

$h_{w m} \quad$ heat transfer coefficient without melting

$k \quad$ thermal conductivity of fluid

$K$ a dimensionless quantity, defined by Eq. (10)

$N \quad$ mass flux

$N_{0} \quad$ mass flux from surface due to melting

$\operatorname{Pr} \quad$ Prandtl number defined as $C_{p \mu} / k$

$q \quad$ heat flux due to conduction

$R_{T} \quad$ dimensionless quantity defined by $\mathrm{Eq}$. (25) or (28)

$t \quad$ time

$T$ temperature

$T_{m} \quad$ melting temperature

$T_{0} \quad$ temperature at surface

$T_{\infty} \quad$ temperature of fluid at infinity

$U \quad$ internal energy per unit mass of fluid

V velocity vector 
$v \quad$ velocity

$v_{\infty} \quad$ velocity of fluid at infinity

$x, y$ coordinates

$\alpha \quad$ thermal diffusivity of fluid

$\Lambda$ dimensionless parameter, equal to unity (with $v$ as subscript) and to $\operatorname{Pr}$ (with $T$ as subscript)

$\begin{array}{ll}\rho & \text { density of fluid } \\ \phi & \text { dimensionless quantity defined by Eq. (38) }\end{array}$

$\phi_{T} \quad$ dimensionless quantity defined by Eq. (26)

$\eta \quad$ dimensionless variable, equal to $(y / 2)\left(v_{\infty} / v x\right)^{\frac{1}{2}}$

$\theta_{T} \quad$ ratio of $h_{m}$ to $h_{w m}$

$\pi \quad$ dimensionless profile for velocity (with $v$ as subscript) or for temperature (with $T$ as subscript)

$\tau \quad$ derivative of $\pi$ with respect to variable $\eta$

$\pi \quad$ stress term of fluid

$\mu \quad$ viscosity

$\nu \quad$ kinematic viscosity

\section{Boundary layer theory}

If the fluid is flowing laminarly over (or under) the solid such as a piece of ice sheet floating in a river, the melting effect or heat transfer can be studied exactly. For a steady-state, two dimensional case, one can write the equations of continuity, motion and energy as follows:

$$
\begin{gathered}
\frac{\partial v_{x}}{\partial x}+\frac{\partial v_{y}}{\partial y}=0 \\
v_{x} \frac{\partial v_{x}}{\partial x}+v_{y} \frac{\partial v_{x}}{\partial y}=\frac{\mu}{\rho} \frac{\partial^{2} v_{x}}{\partial y^{2}} \\
v_{x} \frac{\partial T}{\partial x}+v_{y} \frac{\partial T}{\partial y}=\alpha \frac{\partial^{2} T}{\partial y^{2}}
\end{gathered}
$$

The boundary conditions are $T=T_{0}=T_{m}$ and $v_{x}=0$ at $y=0 ; T \rightarrow T_{\infty}, v_{x} \rightarrow v_{\infty}$ as $y \rightarrow \infty$. The implicit assumptions are (a) existence of boundary layer flow, (b) constant property fluid and (c) no pressure gradient. From the continuity equation, we have

$$
v_{y}=v_{y 0}-\int_{0}^{y} \frac{\partial v_{x}}{\partial x}
$$

The interfacial velocity exists as a result of melting and its magnitude is related to the heat flux transferred from the fluid to the plate as

$$
v_{y^{0}}=k(\partial T / \partial y)_{0} / \rho \Delta H_{m}
$$

or

$$
v_{y}=\frac{k \cdot\left(\frac{\partial T}{\partial y}\right)_{0}}{\rho \cdot \Delta H_{m}}-\int_{0}^{y} \frac{\partial v_{x}}{\partial x} d y .
$$

By introducing dimensionless quantities $\pi_{v}=v_{x} / v_{\infty}$, $\pi_{T}=\left(T-T_{0}\right) /\left(T_{\infty}-T_{0}\right), \Lambda_{v}=1$ and $\Lambda_{T}=\operatorname{Pr}$ Eqs. (2) and (3) can be written into a common form:

$$
\begin{aligned}
v_{x} \frac{\partial \pi}{\partial x}+\left[\frac{C_{p}\left(T_{\infty}-T_{0}\right)}{\Delta H_{m}} \frac{k}{C_{p} \rho}\left(\frac{\partial \pi_{T}}{\partial y}\right)_{0}\right. \\
\left.-\int_{0}^{y} \frac{\partial v_{x}}{\partial x} d y\right] \frac{\partial \pi}{\partial y}=\frac{v}{\Lambda} \frac{\partial^{2} \pi}{\partial y^{2}},
\end{aligned}
$$

where $\pi=0$ at $y=0 ; \pi \rightarrow 1$ as $y \rightarrow \infty$.

Eq. (7) together with the boundary conditions can be solved with the familiar similarity transformation. Assume $\pi$ is a function of $\eta$ defined as $\eta=(y / 2)\left(v_{\infty} / v x\right)^{\frac{1}{2}}$ Eq. (7) becomes

where

$$
\pi^{\prime \prime}+f \Lambda \pi^{\prime}=0
$$

$$
f=\int_{0}^{\eta} 2 \pi_{v} d \eta-K
$$

$$
\begin{aligned}
& K=\frac{1}{\operatorname{Pr}} \frac{C_{p}\left(T_{\infty}-T_{0}\right)}{\Delta H_{m}} \pi_{T^{\prime}}(0, \operatorname{Pr}, K) \\
&=\left(\frac{1}{\operatorname{Pr}}\right)(A) \pi_{T^{\prime}}(0, \operatorname{Pr}, K) .
\end{aligned}
$$

Numerical solutions of $\pi$ for various values of $K$ and $\Lambda$ have been given by a number of investigators. For this work, the interest is focussed on the effect of the interfacial velocity as a result of melting on the heat transfer rate. Let $\theta_{T}$ be the ratio of the heat transfer coefficient with melting to that without melting, we have

$$
\theta_{T}=\frac{h_{m}}{h_{w m}}=\frac{\pi_{T^{\prime}}(0, \operatorname{Pr}, K)}{\pi_{T^{\prime}}(0, \operatorname{Pr}, 0)} .
$$

Numerical values of $K \cdot \operatorname{Pr} / \pi_{T}{ }^{\prime}(0, \operatorname{Pr}, K)$ as a function of $\theta_{T}$ have been compiled by Stewart (1950). From definition of $K$ (see Eq. (10)) one can obtain a relationship between $\theta_{T}$ and $C_{p}\left(T_{\infty}-T_{0}\right) / \Delta H_{m}$ since :

$$
\begin{aligned}
\frac{K \cdot \operatorname{Pr}}{\pi_{T^{\prime}}(0, \operatorname{Pr}, 0)} & =\frac{1}{\operatorname{Pr}} \frac{C_{p}\left(T_{\infty}-T_{0}\right)}{\Delta H_{m}} \pi_{T^{\prime}}(0, \operatorname{Pr}, K) \frac{\operatorname{Pr}}{\pi r^{\prime}(0, \operatorname{Pr}, 0)} \\
& =\frac{C_{p}\left(T_{\infty}-T_{0}\right)}{\Delta H_{m}} \frac{\pi_{T^{\prime}}(0, \operatorname{Pr}, K)}{\pi r^{\prime}(0, \operatorname{Pr}, 0)} \\
A & =\frac{C_{p}\left(T_{\infty}-T_{0}\right)}{\Delta H_{m}}=\left(\frac{K \cdot \operatorname{Pr}}{\pi_{T^{\prime}}(0, \operatorname{Pr}, 0)}\right) / \theta_{T^{\prime}} .
\end{aligned}
$$

\section{Film theory}

The analysis given in the previous section was obtained on the basis of two-dimensional, forced laminar flow. In a practical situation, in addition to laminar flow, one is also interested in the heat transfer between a melting solid and fluid under natural flow or forced 
turbulent flow conditions. For these cases, one can carry out the analogous analysis by solving the proper equation of motion and energy. The resulting equation, however, may become too complicated to be solved analytically. To overcome this difficulty, the effect of melting on heat transfer is to be studied by first proposing a mechanism which is assumed to be valid in describing the transport process between a solid boundary and a surrounding fluid. Since these assumed mechanisms are supposed to be valid under all conditions, the results subsequently obtained will be applicable to a wide category of situations. An analysis on the basis of film theory will be first given in the following paragraphs.

Film theory was first postulated by Whitman (1923) to describe the interphase transport phenomena. It is assumed that the resistance to a transport process between a solid and fluid is confined to a thin layer of stagnant film immediately adjacent to the solid boundary. The model is assumed to be one-dimensional (perpendicular to the surface) and independent of time (steady-state). For energy transport, we have

$$
\nabla \cdot \mathbf{E}=0
$$

where $\mathbf{E}$ is the energy vector. For the one-dimensional case

$$
\mathbf{E}=-k(d T / d y)+N H,
$$

where $N$ is the mass flux and $H$ the enthalpy of fluid at the prevailing temperature. Combining (13) and (14) it follows

$$
-k(d T / d y)+N H=\text { constant. }
$$

Applying Eq. (15) to $y=0$, we have

$$
-k \frac{d T}{d y}+. V H=-\left.k \frac{d T}{d y}\right|_{0}+\aleph_{0} H_{0} .
$$

Because of the one-dimensional dependence, one can show that $N_{0}=N=$ constant, independent of $y$. Eq. (16), after rearrangement becomes

Since

$$
N_{0}\left(H-H_{0}\right)-k \frac{d T}{d y}=-\left.k \frac{d T}{d y}\right|_{0}=q_{0} .
$$

$$
N_{0}\left(H-H_{0}\right)=N_{0} C_{p}\left(T-T_{0}\right) .
$$

Combining (17), (18) we have

$$
N_{0} C_{p}\left(T-T_{0}\right)-k \frac{d T}{d y}=q_{0} .
$$

The boundary conditions are $T=T_{0}$ al $y=0, T=T_{\infty}$ at $y=\delta_{T} . \delta \mathrm{T}$ is the thickness of the film. The solution of (19) is

$$
1-\frac{\left(T-T_{0}\right) C_{p} N_{0}}{q_{0}}=\exp \left(N_{0} C_{p} y / k\right)
$$

or

$$
1-\frac{\left(T_{\infty}-T_{0}\right) C_{p} N_{0}}{q_{0}}=\exp \left(N_{0} C_{p} \delta_{T} / k\right)
$$

The right hand side of Eq. (21) can be written in terms of Taylor's series as

$$
\begin{aligned}
1-\frac{\left(T_{\infty}-T_{0}\right) C_{p} N_{0}}{q_{0}}=1+C_{p} V_{0}^{\gamma} \frac{\delta_{T}}{k} & \\
& +\frac{1}{2 !}\left(C_{p} Y_{0} \frac{\delta_{T}}{k}\right)^{2}+\cdots
\end{aligned}
$$

Consider the limiting case where there is no melting $N_{0} \rightarrow 0$. From (22) we have

$$
\frac{\delta_{T}}{k}=\lim _{N_{0} \rightarrow 0} \frac{-\left(T_{\infty}-T_{0}\right) / q_{0}}{N_{0}}=\frac{1}{h_{w m}},
$$

where $h_{w m}$ is the local heat transfer coefficient without melting. It should be noted that $q_{0}$ is inherently negative since the heat is transferred from fluid to plate.

Combining (21), (23), one has

$$
1+\frac{\left(T_{\infty}-T_{0}\right) C_{p} Y_{0}}{-q_{0}}=\exp \left(C_{p} \Lambda_{0} / h_{w m}\right)
$$

If one introduces the following quantities

$$
\begin{aligned}
R_{T} & =\frac{\left(T_{\infty}-T_{0}\right) C_{p} N_{0}}{-q_{0}}=\frac{C_{p} N_{0}}{h_{m}} \\
\phi_{T} & =\frac{C_{p} N_{0}}{h_{w m}} \\
\theta_{T} & =\frac{h_{m}}{h_{w m}}=\frac{\phi_{T}}{R_{T}}
\end{aligned}
$$

also

$$
N_{0}^{T}=k\left(\frac{d T}{d y}\right)_{0} / \Delta H_{m}=-q_{0} / \Delta H_{m}
$$

therefore

$$
R_{T}=\frac{\left(T_{\infty}-T_{0}\right) C_{p}}{-q_{0}} \frac{-q_{0}}{\Delta H_{m}}=A .
$$

Combining Eqs. (24)-(28), we have $1+R_{T}=\exp \left(\phi_{T}\right)$, or $\phi_{T}=\ln \left(1+R_{T}\right)$ and

$$
\theta_{T}=\frac{\phi_{T}}{R_{T}}=\frac{\ln (1+A)}{A} .
$$

Eq. (29) expresses the effect of melting on heat transfer coefficient in terms of the parameter $A$.

\section{Penetration theory}

We now proceed to examine the effect of melting on heat transfer on the basis of penetration theory which 
was first proposed by Higbie (1935) and modified and expanded by many other investigators, (Danckwerts, 1951; Hanratty, 1956; Toor and Marchello, 1958; Perlmutter, 1961) in recent years. Based upon experimental evidence on mass transfer, it appears that this theory provides a more plausible explanation for interphase transport processes (energy and mass). It assumes that the transport process is effected by the sweeping of small eddies of the fluid in a turbulent field into contact with the interfaces. Since the contact time, for most cases, is small, the eddy may be treated as a semi-infinite solid and a transient state heat conduction equation can be used in describing the energy transfer. Although one cannot predict the transfer coefficient based on this model unless one has the complete knowledge of the surface renewal mechanism (or in other words, the residence-time distribution of eddy), it is possible to study the effect of interfacial velocity resulting from melting on heat transfer if one is to assume that the surface renewal mechanism is not affected by melting.

The unsteady state energy equation is written to describe the eddy. Namely,

$$
\begin{gathered}
\frac{\partial}{\partial t} \rho\left(U+\frac{1}{2} v^{2}\right)=-\nabla \cdot \mathbf{E} \\
E=\rho\left(U+\frac{1}{2} v^{2}\right) \mathbf{V}-k \nabla T+(\tau \cdot \mathbf{V}) .
\end{gathered}
$$

For a one dimensional case, neglect the kinetic energy term and $\tau \cdot \boldsymbol{V}$. For constant property, we have

$$
\rho \frac{\partial U}{\partial t}=-\frac{\partial}{\partial t}(X U)+k \frac{\partial^{2} T}{\partial y^{2}} \text {. }
$$

As in the case of film model, penetration also presuppose unidirectional dependence, this implies that

and furthermore

$$
N=\text { Constant }=\nu_{0}=k\left(\frac{\partial T}{\partial y}\right)_{0} / \Delta H_{m}
$$

$$
U=C_{p}\left(T-T_{r}\right),
$$

$T_{r}$ being any reference temperature. Combining (32)(34), it follows

$$
\frac{\partial T}{\partial t}=\alpha \frac{\partial^{2} T}{\partial y^{2}}-\frac{\alpha C_{p}\left(\frac{\partial T}{\partial y}\right)_{0}}{\Delta H_{m}} \frac{\partial T}{\partial y} .
$$

The boundary and initial conditions are $T=T_{\infty}$ at $y \geqslant 0, t \leqslant 0 ; T=T_{0}$ at $y=0, t \geqslant 0$ and $T \rightarrow T_{\infty}$ as $y \rightarrow \infty$. Solution to $(35)$ is

$$
T^{+}=\frac{T-T_{\infty}}{T_{0}-T_{\infty}}=\frac{1-\operatorname{erf}(\beta+\phi)}{1-\operatorname{erf} \phi}
$$

where

$$
\begin{aligned}
& \beta=y /(4 \alpha t)^{\frac{1}{2}} \\
& \phi=(A / 2)\left(T^{+}\right)_{0}^{\prime} .
\end{aligned}
$$

Differentiating (36) and substituting it into (38), it follows that

$$
(1-\operatorname{erf} \phi) \sqrt{\pi} \phi \exp \left(\phi^{2}\right)=-A
$$

The heat flux $q_{0}$ is

$$
\begin{aligned}
q_{0} & =-k\left(\frac{\partial T}{\partial y}\right)_{0}=-k\left(T_{0}-T_{\infty}\right)\left(\frac{\partial T^{+}}{\partial y}\right)_{0} \\
& =k\left(T_{\infty}-T_{0}\right)\left(T^{+}\right)_{0}\left(1 /(4 \alpha t)^{\frac{1}{2}}\right) \\
& =k\left(T_{\infty}-T_{0}\right)\left(\frac{-1}{1-\operatorname{erf} \phi}\right) \frac{2}{\sqrt{\pi}} \frac{1}{\sqrt{4 \alpha} t} \exp \left(-\phi^{2}\right) .
\end{aligned}
$$

The transfer coefficient, $h_{m}$ is defined as

$$
h_{m}=\frac{-q_{0}}{T_{\infty}-T_{0}}=\frac{k \exp \left(-\phi^{2}\right)}{(1-\operatorname{erf} \phi) \sqrt{4 \alpha t}}
$$

and the limiting value of $h_{m}$ for no melting is obtained by taking the limit of $h_{m}$ for $\phi \rightarrow 0$. This gives

$$
h_{w m}=\lim _{\phi \rightarrow 0} h_{m}=\frac{k}{\sqrt{4 \alpha t}} .
$$

The ratio of $h_{m} / h_{w m}$ is thus

$$
\theta_{T}=\frac{h_{m}}{h_{w m}}=\frac{1}{(1-\operatorname{erf} \phi) \exp \left(\phi^{2}\right)} .
$$

The effect of melting on heat transfer coefficient can be seen by combining Eqs. (39) and (43).

\section{Conclusion}

The effect of melting on heat transfer as expressed in terms of $\theta_{T}=h_{m} / h_{w m}$ versus the parameter $A=C_{p} \Delta T$ / $\Delta H_{m}$ describing the thermal state has been studied in the investigation for three different models. The results are given in Table 1 and also shown graphically in Fig. 1. Also included are the results obtained earlier by Yen and Tien (1963) on the model of Leveque problem. Merk (1954) studied analytically the effect of melting on heat transfer by solving the equations of motion and energy using the approximate integral method. The final expression in our notation is

$$
\theta_{T}=\frac{h_{m}}{h_{w m}}=\left[\frac{1-\frac{89}{217} s+\frac{19}{434} s^{2}}{1-\frac{1}{2} s+\frac{1}{4} s^{2}}\left(1+\frac{1}{2} s\right)^{5}\right]^{\frac{1}{4}}
$$


TABLE 1. Effect of parameter $A$ on heat transfer rate.

\begin{tabular}{cccccc}
\hline$A=\frac{C_{p}\left(T_{\infty}-T_{0}\right)}{\Delta H_{m}}$ & $\begin{array}{c}\text { Boundary layer } \\
\text { theory }\end{array}$ & Film theory & $\begin{array}{c}\theta_{T}=h_{n} / h_{w m} \\
\text { Penetration } \\
\text { theory }\end{array}$ & Merk (1954) & $\begin{array}{c}\text { Yen and Tien } \\
(1963)\end{array}$ \\
\hline 0.01006 & 0.9940 & 0.9950 & 0.9943 & 0.9956 & 0.990 \\
0.02022 & 0.9890 & 0.990 & 0.9887 & 0.9915 & 0.986 \\
0.05144 & 0.9720 & 0.9751 & 0.9713 & 0.9785 & 0.966 \\
0.10593 & 0.9440 & 0.9505 & 0.9428 & 0.9573 & 0.927 \\
0.22472 & 0.8900 & 0.9021 & 0.8879 & 0.9156 & 0.858 \\
0.67659 & 0.5390 & 0.7638 & 0.7219 & 0.7964 & 0.668 \\
1.90840 & 0.2300 & 0.5594 & 0.5009 & 0.6177 & - \\
8.69565 & 0.0830 & 0.2612 & 0.2053 & 0.3395 & - \\
36.14457 & 0.0061 & 0.0082 & 0.0703 & 0.1637 & - \\
815.66089 & & & 0.0040 & 0.0263 & - \\
\hline
\end{tabular}

in which $s$ is given in terms of $A$ by

$$
s=-2-\frac{3}{A}+\frac{3}{A}\left(1+\frac{4}{3} A\right)^{\frac{1}{2}} .
$$

Values of $\theta_{T}$ as function of $A$ computed from the above two expressions are also shown in Table 1 and Fig. 1 for comparison. Although the quantitative results differ among each other, they all show the same qualitative trend and indicate that melting inhibits the heat transfer rate. The results based on Leveque solution agree with those based on boundary layer theory for

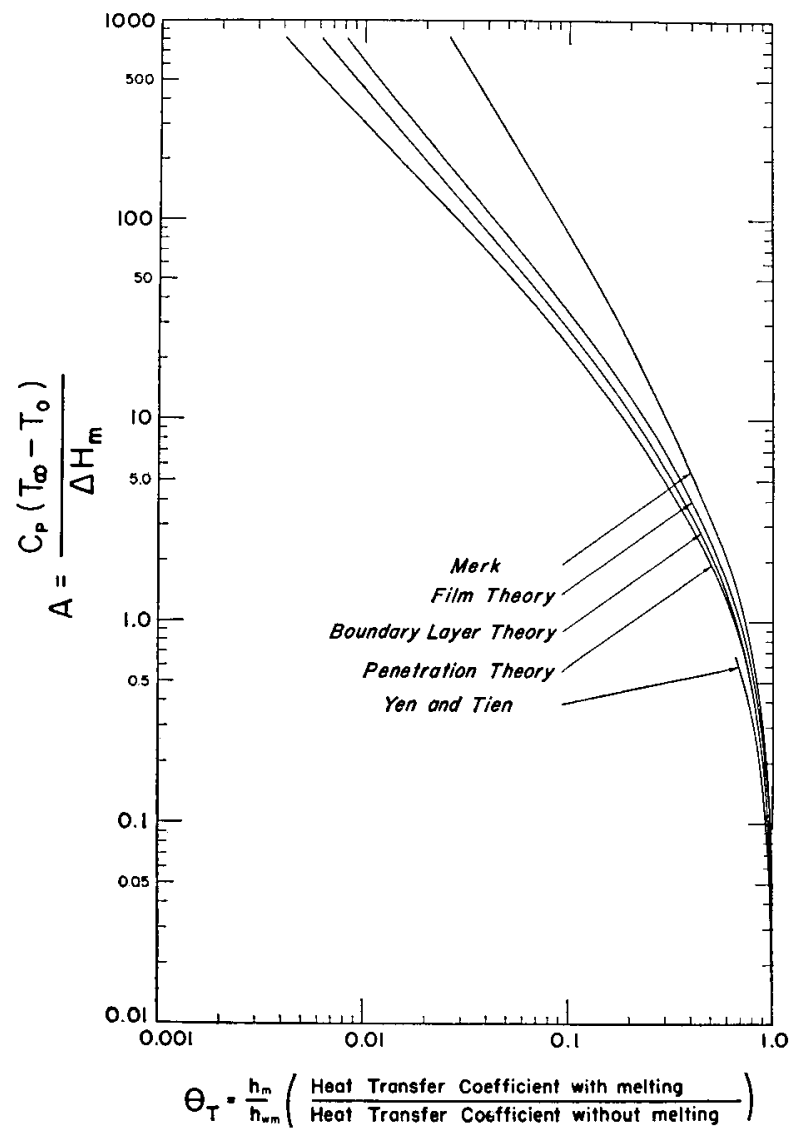

FIG. 1. Relationship between parameters $A$ and $\theta_{T}$. small values of $A$ but deviate from each other as " $A$ " increases. This is expected since the solution obtained by Yen and Tien, in a sense, is an asymptotic solution of that based on boundary layer theory for small $A$. The difference in results based on these three models makes it necessary that caution should be exercised on the selection among these results for practical application. This will, to a large extent, depend upon the flow condition. For example, if the flow is laminar, the results based on boundary layer theory should certainly be used. On the other hand, if the flow is turbulent (such as the case where either the longitudinal dimensions of melting solid is large or the velocity of the fluid is high) or in cases where natural flow or a combination of forced and natural flow exists, no definite conclusion can be made as to which of these results should be used. It is suspected that the results based on penetration theory should provide a better picture because it has been verified experimentally to be more adequate in explaining the related transport process of mass transfer. This, however, should be strictly considered as a speculation until more experimental results become available in this field.

\section{REFERENCES}

Bird, R. B., W. E. Stewart and E. N. Lightfoot, 1960: Transport Phenomena, New York, John Wiley and Sons, Inc., 613 pp.

Danckwerts, P. V., 1951: Significance of liquid film coefficients in gas absorption. Ind. Eng. Chem., 43, 1460-1467.

Hanratty, T. J., 1956: Turbulent exchange of mass and momentum with a boundary. Amer. Inst. Chem. Eng., 2, 359-362.

Higbie, R., 1935: The rate of absorption of a pure gas into a still liquid during short periods of exposure. Trans. Amer. Inst. Chem. Eng., 31, 365-389.

Merk, J. H., 1954: The influence of melting and anomalous expansion on the thermal convection in laminar boundary layers. A ppl. Sci. Res., 4, section A, 435-452.

Perlmutter, D. D., 1961: Surface renewal models in mass transfer. Cliem. Eng. Sci. 16, 287-296.

Stewart, W. E., 1950: Interaction of heat, mass and momentum transfer. D.Sc. Thesis, Mass. Inst. Tech., Cambridge, Mass.

Toor, H. L., and J. M. Marchello, 1958: Film penetration model for mass and heat transfer. Amer. Inst. Chem. Eng., 4, 97-101.

Whitman, W. G., 1923: Chem. Melall. Eng., 24, 146.

Yen, Y. C., and C. Tien, 1963: Laminar heat transfer over a meiting plate, the modified Leveque problem. J. Geophys. Res., $68,3673-3678$. 